\title{
Influencia del rolado selectivo de baja intensidad (RBI) sobre las comunidades de aves de bosques del Chaco Occidental
}

\author{
RubÉn D. Coria ${ }^{1, \bowtie}$; OsCAR R. CORIA ${ }^{2} \&$ CARLOS R. KunST ${ }^{1}$ \\ 1. Instituto Nacional de Tecnología Agropecuaria, EEA Santiago del Estero. 2. Facultad de Ciencias Forestales, Universidad \\ Nacional de Santiago del Estero (UNSE).
}

\begin{abstract}
RESUMEN. El rolado selectivo de baja intensidad (RBI) es una tecnología de habilitación de sistemas silvopastoriles de bajo impacto usado en la Región Chaqueña. El RBI reduce el estrato leñoso bajo ( $<3 \mathrm{~m}$ de altura), incrementa la cobertura herbácea y puede aplicarse en combinación con la corta forestal. El objetivo de este estudio fue evaluar a escala local la influencia de dos bosques tratados con RBI de diferentes edades (uno y siete años atrás) sobre las diversidades alfa y beta y la abundancia de los gremios tróficos de aves de bosques del Chaco Occidental en las épocas reproductiva y no reproductiva. Respecto al bosque de referencia sin disturbar, los resultados sugieren que: i) el RBI generó hábitats de bajo contraste, ii) el tratamiento de mayor edad fue el menos contrastado, iii) la diversidad alfa no disminuyó en respuesta a los tratamientos y la diversidad beta fue baja en ambas épocas, y iv) el tratamiento de menor edad tuvo la composición más diferente del ensamble de aves dado que en ambas épocas tuvo la mayor diversidad beta y presentó modificaciones en la abundancia de los gremios, asociadas al RBI. Este estudio sugiere que: i) los bosques tratados con RBI mantienen condiciones de hábitat y composiciones de ensambles de aves similares a los bosques sin disturbar, en particular en los tratamientos de mayor edad, y ii) el RBI presenta potencial para satisfacer objetivos de conservación de aves y producción sustentable.
\end{abstract}

[Palabras clave: sistemas silvopastoriles, diversidad alfa, diversidad beta, gremios tróficos, vegetación]

\begin{abstract}
AвSTRACT. Influence of low intensity roller chopping (RBI) on bird communities in the Western Chaco forests. Low intensity roller-chopping (RBI) is a technology applied to develop low impact silvopastoral systems in the Chaco Region. The RBI reduces the lower woody stratum $(<3 \mathrm{~m}$ high) and increases the herbaceous cover, and it can be applied in combination with partial cuts of the forest. The aim of this study was to evaluate the influence at the local scale of two RBI treatments of different age (after one and seven years) on alpha and beta diversity and the trophic guild structure of forest birds in the Western Chaco, in the breeding and non-breeding seasons. Results suggest that, compared to undisturbed forest: i) the RBI created habitats of low contrast, ii) the older treatment was the less contrasted, iii) alpha diversity did not decrease in response to treatments and both seasons had low beta diversity, and iv) recent treatment had the greatest dissimilarity in bird assemblage composition, since both seasons had the highest beta diversity and bigger changes in abundance of the guilds associated with RBI. This study suggest that: i) forests treated whit RBI maintain habitat conditions and compositions of bird assemblages similar to the undisturbed forest, particularly in older treatments, and ii) RBI has potential to meet simultaneously the goals of bird conservation and sustainable production.
\end{abstract}

[Keywords: silvopastoral systems, alpha diversity, beta diversity, trophic guilds, vegetation]

\section{INTRODUCCIÓN}

El Chaco Occidental con clima semiárido es una subregión del Gran Chaco Sudamericano, cuya vegetación original se componía principalmente de un paisaje de bosques xerófilos y sabanas (Morello \& Adamoli 1968). A partir del siglo XX, la intensificación del uso ganadero y forestal tuvo como consecuencia la lignificación (predominio de leñosas, en particular arbustivas) en detrimento de las coberturas herbáceas de bosques y sabanas (Morello \& Saravia Toledo 1959; Bucher 1980; Kunst et al. 2008). En respuesta a esta problemática, diversos disturbios fueron utilizados para controlar las leñosas (e.g., remoción mecánica, manual, química y con fuegos) e incrementar la productividad de

Editor asociado: Fernando Milesi

$\triangle$ rudacoria@yahoo.com.ar forraje herbáceo, especialmente con la siembra de pasturas exóticas. En bosques, la remoción de leñosas con fines ganaderos genera sistemas silvopastoriles (SSPs) y sistemas pastoriles (SPs). Según la intensidad de los disturbios, estos sistemas se clasifican en SSPs de baja de intensidad (bosques abiertos con remoción parcial de arbustos), SSPs de intensidad intermedia (árboles nativos dispersos donde el sotobosque ha sido reemplazado por pasturas exóticas) y SPs de alta intensidad (reemplazo total de leñosas por pasturas exóticas) (Mastrangelo \& Gavin 2012). Recientemente se desarrolló una tecnología de habilitación de SSPs de baja intensidad denominada rolado selectivo de baja intensidad (RBI) con el fin de integrar la producción ganadera y

Recibido: 24 de noviembre de 2014, Fin de arbitraje: 5 de febrero de 2015, Última versión revisada: 5 de junio de 2015, Aceptado: 5 de junio de 2015 
forestal sustentable (Kunst et al. 2008). Esta práctica reduce el estrato leñoso bajo $(<3 \mathrm{~m}$ de altura, principalmente plantas arbustivas), incrementa la cobertura herbácea (en especial con la siembra de pasturas exóticas) y puede aplicarse en combinación con la corta forestal (remanente de área basal de árboles $>6 \mathrm{~m}^{2} / \mathrm{ha}$ ). El RBI se realiza con rolo, un cilindro metálico de $2.5 \mathrm{~m}$ de ancho armado con cuchillas que, traccionado por maquinaria agrícola pequeña, aplasta y tritura las leñosas a su paso, pero no las mata (Albanesi et al. 2013).

En el Chaco Occidental, los estudios de la influencia de los SSPs y SPs sobre la fauna son escasos y con énfasis en las aves. En un paisaje con fragmentos de bosques, corredores con remanentes de bosque que conectan los fragmentos y SPs de alta intensidad hubo gran uniformidad en la riqueza de mamíferos, reptiles y anfibios entre los tres componentes del paisaje, con excepción de la riqueza de aves que disminuyó en los SPs (Areskoug 2001). Durante el verano, la riqueza y abundancia de aves disminuyó en un SSP de siete años de antigüedad, desarbustado de forma manual (Codesido et al. 2009). En un gradiente de intensificación de la producción ganadera (i.e., fragmentos de bosques< $<$ sistemas de muy baja intensidad $<$ SSPs de baja intensidad $<$ SSPs de intensidad intermedia $<$ SPs de alta intensidad) la riqueza de aves decreció a lo largo del gradiente de manera no lineal (Mastrangelo \& Gavin 2012). En otro gradiente de intensificación productiva (i.e., bosques protegidos $<$ puestos ganaderos extensivos $<S$ SPs $<$ SPs $<$ cultivos de soja) la riqueza de aves decreció abruptamente en SPs y cultivos de soja, pero la abundancia decayó de forma monótona a lo largo del gradiente (Macchi et al. 2013). El primer estudio de un bosque tratado con RBI de dos años de antigüedad mostró que la diversidad alfa de aves no se modificó (Coria et al. 2012). La mayoría de estos estudios reportaron variaciones en las estructuras de gremios tróficos de aves asociados a los cambios en la vegetación y los hábitos de las especies. Esta revisión indica que la diversidad de aves disminuye con el incremento de la intensidad del disturbio, por lo cual los SSPs de baja intensidad son deseables desde la perspectiva de la conservación. Si bien Coria et al. (2012) evaluaron las respuestas de la diversidad alfa y estructura gremial de las aves en un bosque tratado con RBI, no contemplaron otros factores como los efectos locales o las diferentes edades de los tratamientos.
La calidad de la matriz como hábitat depende de la similaridad estructural (o contraste) entre la matriz y los parches de vegetación (Fischer et al. 2006). En el Chaco Occidental, cuanto menos contrastados son los parches de bosque con las matrices productivas ganaderas más similares son las composiciones de los ensambles de aves (Mastrangelo \& Gavin 2012). El RBI provoca bajo impacto en la vegetación y su efecto principal es la generación de bosques parcialmente abiertos que tienden a cerrarse con el paso del tiempo, en especial por la recuperación de la cobertura arbustiva (Kunst et al. 2008; Albanesi et al. 2013), por lo que se espera que el grado de contraste varíe con la edad de los tratamientos.

El objetivo de este estudio fue evaluar a escala local la influencia de dos bosques tratados con RBI de diferentes edades (después de uno y siete años) sobre las diversidades alfa y beta y sobre los gremios tróficos de aves en bosques del Chaco Occidental, tanto en la época reproductiva como no reproductiva. Abordamos las siguientes hipótesis $(\mathrm{H})$ y predicciones (p). (H1) El bajo impacto del RBI sobre la vegetación permite mantener la calidad del bosque como hábitat para su avifauna. Si el RBI es una tecnología de bajo impacto que no modifica sustancialmente la estructura y composición de la vegetación, se espera que entre el bosque de referencia y los tratados con RBI exista: (p1.1) un contraste de hábitat bajo, (p1.2) diversidades alfa sin disminuciones significativas y (p1.3) diversidades beta bajas. (H2) La edad de los tratamientos con RBI determina el grado de contraste entre los bosques tratados y el de referencia. Si la vegetación afectada por el RBI se recupera con el tiempo, se espera que (p2) el grado de contraste con el bosque de referencia disminuya con la edad del tratamiento. (H3) El grado de contraste entre los hábitats determina el grado de similitud entre los ensambles de aves. Si las aves responden a las características diferenciales entre los hábitats reflejados en los distintos grados de contraste, se espera que entre los bosques más contrastados (p3.1) la diversidad beta de aves sea mayor y (p3.2) las estructuras de gremios tróficos presenten las mayores diferencias.

\section{MATERIALES Y MÉTODOS}

\section{Área de estudio}

El área de estudio es el Campo Experimental La María $\left(28^{\circ} 3^{\prime}\right.$ S, $64^{\circ} 15^{\prime}$ O) del INTÁ, Estación Experimental Agropecuaria Santiago del 
Estero. El clima es semiárido subtropical con estacionalidad bien marcada (Boletta 1998). La temperatura promedio anual es $21^{\circ} \mathrm{C}$, con una mínima y una máxima promedio de $14{ }^{\circ} \mathrm{C}$ y $28^{\circ} \mathrm{C}$, respectivamente. La precipitación media es 574 $\mathrm{mm} / \mathrm{año}$, concentrada en los meses de octubre a marzo. Enclavado en el Chaco Occidental (Bucher 1982), el Campo La María posee una matriz de vegetación nativa continua de $60 \mathrm{~km}^{2}$, integrada por parches de bosques, fachinales y sabanas. Los árboles dominantes en los bosques son Aspidosperma quebracho-blanco y Schinopsis lorentzii, seguidos por Ziziphus mistol y Prosopis nigra. Entre los arbustos dominan Acacia gilliesii, Capparis atamisquea y Celtis ehrenbergiana, con menor participación de Moya spinosa. En el estrato herbáceo son frecuentes las gramíneas Gouinia latifolia, Gounia paraguayensis, Setaria lachnea, Setaria sp. y Trichloris crinita, y entre las latifoliadas, Justicia squarrosa, Wissadula densiflora y Amphilophium carolinae.

\section{Tratamientos}

Los tratamientos (tipos de bosques) fueron tres: 1) bosque testigo: bosque con ganadería y sin aprovechamiento forestal en tres décadas, 2) RBI antiguo: bosque de 32 ha tratado con RBI de siete años de antigüedad, con siembra de Megathyrsus maximus co gatton panic (densidad de siembra $=6$ $\mathrm{kg} / \mathrm{ha}$ ), con pastoreo ganadero y corta forestal (30\% del área basal extraída), y 3) RBI reciente: bosque de 100 ha tratado con RBI de un año de antigüedad, sin siembra de pasturas exóticas, con pastoreo ganadero y corta forestal $(30 \%$ del área basal apeada, pero extraída sólo en un $8.2 \%$ ).

Los tres tipos de bosques integraron un gran bloque de vegetación nativa de aproximadamente $4 \times 16 \mathrm{~km}$. El bosque testigo y el RBI antiguo se hallaban próximos $(665 \mathrm{~m}$ desde el centro al borde) a un campo vecino con SSPs de intensidad intermedia y SPs de alta intensidad. El RBI reciente se hallaba próximo ( $820 \mathrm{~m}$ desde el centro al borde) a un campo vecino dividido en parcelas de cultivo de 50 ha rodeadas con cortinas de vegetación nativa de $50 \mathrm{~m}$ de ancho en promedio. Los bosques estudiados estuvieron sometidos a dos tipos de influencias en el contexto de paisaje: el gran bloque de vegetación nativa con cobertura leñosa densa y, en menor medida, los hábitat abiertos donde gran parte de la vegetación leñosa fue removida (campos productivos vecinos).

\section{Muestreo de aves}

Se utilizó el método de conteo por puntos de radio ilimitado, con detección visual y auditiva de individuos de cada especie durante $10 \mathrm{~min} /$ punto (Gibbons et al. 1996). El radio ilimitado permite mayor detectabilidad de especies raras o escasas en relación al radio fijo (Codesido \& Bilenca 2000). Los muestreos se hicieron siempre por la mañana, entre las 6:45 h y las 9:30 h. No fueron consideradas las aves que sobrevolaban por encima de los $20 \mathrm{~m}$
(Codesido \& Bilenca 2004). En bosque testigo y RBI reciente se ubicaron siete estaciones de muestreo a 200-300 m de los bordes de caminos internos para minimizar los efectos de borde. En RBI antiguo, dado su menor superficie, se dispusieron cinco estaciones de muestreo a no menos de $100 \mathrm{~m}$ de tales bordes. Para reducir las posibilidades de contar más de una vez a los mismos individuos, las estaciones de muestreo se ubicaron a más de $250 \mathrm{~m}$ entre sí (la distancia mínima recomendada es $200 \mathrm{~m}$ ) (Gibbons et al. 1996). En cada estación de muestreo se realizaron dos mediciones de las aves, una en la época no reproductiva - a comienzos de septiembre de 2013 - y otra en la época reproductiva, entre fines de octubre y comienzos de noviembre del mismo año. Debido a que comienzos de septiembre estuvo muy próximo al inicio de la época reproductiva, se corroboró al momento de la toma de datos que efectivamente las aves no habían comenzado con la reproducción, y se verificó que: i) la comunidad de aves estuvo compuesta principalmente por especies residentes, con ausencia total de especies migrantes estivales neotropicales que marcan la presencia de la época reproductiva, y ii) la inexistencia de comportamiento reproductivo (establecimiento de territorios y perchas para cantar, competencia entre machos, nidificación). En la nomenclatura de especies de aves se siguió a Remsen et al. (2014).

En septiembre del año 2009 se realizó un estudio para evaluar si la metodología descripta produce sobreestimaciones de aves debido al incremento de la visibilidad en bosques tratados con RBI. En cada uno de dos bosques aledaños, uno sin tratar y otro tratado con RBI (de dos años de antigüedad), se ubicaron seis estaciones de muestreo con distancias de $150 \mathrm{~m}$ entre sí y a más de $100 \mathrm{~m}$ de los bordes. En un lapso de un día y medio se realizaron tres mediciones por estación de muestreo, consignando las detecciones visuales y auditivas por separado. Las detecciones visuales se promediaron por estación demuestreo y luego se obtuvola proporción de individuos vistos (i.e., promedio de 0.16 por cada tipo de bosque), que no difirió entre los dos bosques (prueba $t$ para muestras independientes luego de la transformación arcoseno, $\mathrm{t}_{10}=0.13, P>0.05$ ). Esto sugiere que no se producen sobrestimaciones por la mayor visibilidad en bosques tratados con RBI.

\section{Muestreo de vegetación}

Los bosques estudiados presentaron heterogeneidad interna a escala de lote. En cada uno de ellos los arbustos se distribuyeron de manera uniforme, pero los árboles más grandes mostraron una tendencia marcada a la agregación y dieron por resultado dos zonas bien definidas: i) con cobertura arbustiva abundante y arbórea escasa (en adelante "arreglo arbustal"), y ii) con coberturas arbustiva y arbórea abundantes (en adelante "arreglo arbolado"). Estos arreglos fueron irregulares, discontinuos, entremezclados y relativamente pequeños (i.e., 0.06-0.24 ha), y tuvieron implicancias en los muestreos de vegetación. 
En cada bosque se midieron las coberturas arbórea, arbustiva, herbácea, de hojarasca y de residuos leñosos y las frecuencias de las especies. La cobertura arbórea estuvo determinada por especies arbóreas de más de $3 \mathrm{~m}$ de altura, la cobertura arbustiva por especies arbóreas y arbustivas de entre 0.5 y 3 m de altura y la cobertura herbácea por gramíneas, latifoliadas herbáceas y juveniles de árboles y arbustos ( $<0.5 \mathrm{~m}$ de altura). Los residuos leñosos fueron troncos, ramas y ramitas muertas mayores a $2 \mathrm{~mm}$ de diámetro, dispuestos en el suelo. La hojarasca se compuso de follaje caído, restos de herbáceas, musgos y ramitas muy finas ( $<2 \mathrm{~mm}$ de diámetro). La evaluación de frecuencias florísticas fue estrictamente taxonómica, sin importar el tamaño de los individuos. Los métodos utilizados fueron: i) transecta de puntos para medición de cobertura de leñosas (se utilizaron transectas de $45 \mathrm{~m}$ y se relevaron 30 puntos en cada una) (Riney 1982), ii) método del vecino más próximo para medición de frecuencias de especies leñosas (Bonham 1989), y iii) estimación visual en marco metálico de 0.25 $\mathrm{m}^{2}$ de cobertura herbácea, hojarasca, residuos leñosos y frecuencias de especies herbáceas. La nomenclatura de especies siguió al Catálogo del Plantas Vasculares de la República Argentina (Instituto de Botánica Darwinion 2014).

Las estaciones de muestreo de vegetación fueron las mismas que las de aves. Sin embargo, para realizar una caracterización más precisa de la vegetación se tomaron muestras discriminadas por arreglo de vegetación. Cada estación de muestreo fue dividida en dos subestaciones de muestreo, una situada en el arreglo arbolado y otra en el arreglo arbustal. Esto dio un total de 14 subestaciones de muestreo en bosque testigo, idéntica cantidad en $\mathrm{RBI}$ reciente y 10 en $\mathrm{RBI}$ antiguo. Entre agosto y septiembre del año 2013 se tomaron tres medidas de cada variable en cada subestación de muestreo, a excepción de la cobertura de leñosas que se midió solo una vez. Durante el lapso en que transcurrió el trabajo de campo (agosto-noviembre de 2013), la región atravesaba una sequía de dos años y se asumió que no hubo cambios significativos en la vegetación por falta de agua. Por lo tanto, los mismos datos de vegetación se utilizaron en los análisis tanto de la época reproductiva como no reproductiva de las aves.

\section{Análisis de datos}

El número de individuos por especie de ave se empleó para estimar las abundancias relativas de cada gremio trófico y la diversidad alfa (i.e., índice Shannon-Weaver). Para evaluar la exhaustividad del muestreo se construyeron curvas de acumulación de especies (Martella et al. 2012). Se definieron 11 gremios tróficos siguiendo a Codesido \& Bilenca (2004) y otras referencias (Biondi et al. 2005; Giraudo et al. 2006; Galmes et al. 2008; Macchi et al. 2013): 1) omnívoras: se alimentan de insectos, semillas y frutos, 2) granívoras terrestres: se alimentan de semillas obtenidas del suelo o de plantas herbáceas a baja altura, 3) granívoras arbóreas: se alimentan de semillas sobre la vegetación leñosa, arbustiva o arbórea, 4) nectarívoras: se alimentan principalmente del néctar de las flores $\mathrm{y}$, en segundo lugar, de insectos, 5) insectívoras de corteza: se alimentan de insectos obtenidos bajo de la corteza de leñosas, 6) insectívoras terrestres: se alimentan de insectos del suelo, 7) insectívoras de follaje: se alimentan de insectos obtenidos de las ramas y hojas, 8) insectívoras de vuelo: se alimentan de insectos en el aire, 9) frugívoras: se alimentan fundamentalmente de la pulpa de frutos de algunas leñosas y secundariamente de insectos, 10) insectívoras carroñeras: se alimentan principalmente de insectos y, en segundo lugar, de carroña, y 11) carnívoras insectívoras: se alimentan principalmente de pequeños vertebrados terrestres y complementan su dieta con insectos.

Para evaluar los cambios en las abundancias gremiales y en la diversidad alfa de aves (variables dependientes) se utilizó análisis multivariado de la varianza de un factor de clasificación (tipo de bosque) con enfoque de medidas repetidas en el tiempo (factor época: reproductiva y no reproductiva). Se utilizó el estadístico de Wilks como base de los análisis y la prueba de Hotelling para comparaciones a posteriori. Para aquellos gremios presentes solo en la época reproductiva (i.e., nectarívoras y frugívoras) se utilizó análisis univariado de la varianza de un factor de clasificación (tipo de bosque). Se utilizó la prueba de Tukey para comparaciones a posteriori. En todas las pruebas multivariadas y univariadas se estableció $\square=0.05$. Se aplicó la transformación raíz cuadrada a los datos para poder sostener los supuestos de normalidad y homogeneidad de varianzas de los residuos (Zar 1999).

Para evaluar la diversidad beta de aves se aplicó el análisis T1 de Anderson et al. (2011), que es una medida del recambio de especies entre dos comunidades. Se utilizó el índice de disimilitud cualitativo de Jaccard $(\mathrm{Jd}=1-\mathrm{Js})$, siendo Js $=\mathrm{C} /(\mathrm{a}+\mathrm{b}-$ $\mathrm{C})$, donde $\mathrm{a}=$ número de especies en el hábitat $\mathrm{A}$, $\mathrm{b}=$ número de especies en el hábitat $\mathrm{B}, \mathrm{y} \mathrm{C}=$ número de especies en ambos hábitats. El intervalo de valores posibles de Js es $0-1$, con 0 cuando no hay especies compartidas entre ambos sitios y 1 cuando los dos sitios tienen la misma composición de especies (Moreno 2001), al revés que para Jd. Se clasificó la diversidad beta de acuerdo a Jd según Coria (2014) como: muy baja (Jd=0-0.09), baja $(\mathrm{Jd}=0.10-0.39)$, media $(\mathrm{Jd}=0.40-0.59)$, alta $(\mathrm{Jd}=0.6-$ $0.89)$ y muy alta $(\mathrm{Jd}=0.90-1)$.

Se analizaron ocho variables de vegetación: coberturas (\%) arbórea, arbustiva, herbácea, de hojarasca y de residuos leñosos, y diversidades alfa de árboles, de arbustos y de herbáceas (índices Shannon-Weaver). Por cada subestación de muestreo: i) las coberturas de árboles y arbustos se obtuvieron mediante $n \times 100 / 30$, donde $n$ es el número de toques de copas y 30 el número total de puntos por transecta (Riney 1982) y, ii) las 
frecuencias vegetales por especie se obtuvieron mediante $n \times 100 / 3$, donde $n$ es el número de veces en que una determinada especie ocupó una submuestra y 3 es el total de submuestras (Bonham 1989). Las frecuencias vegetales se emplearon como estimadoras de la diversidad alfa de especies. Los datos de cobertura herbácea y de residuos leñosos fueron los recolectados directamente a campo.

Para evaluar los cambios en las variables de vegetación (variables dependientes) se utilizó análisis dela varianza de dosfactores declasificación: tipo de bosque y arreglo de vegetación. Se utilizó la prueba de Tukey para comparaciones a posteriori, estableciendo $\square=0.05$. Se aplicó la transformación raíz cuadrada a los datos para poder sostener los supuestos de normalidad y homogeneidad de varianzas de los residuos (Zar 1999).

El análisis de correspondencia sin tendencias ("DCA", del inglés detrended correspondence analysis) (Hill \& Gauch 1980) fue utilizado para evaluar el grado de contraste de hábitat considerando todas las variables de vegetación. El DCA es un tipo de análisis multivariado indirecto que permite visualizar patrones en gráficos de ordenamientos en base a medidas de similitudes. Logra un ordenamiento que es independiente de nociones preconcebidas acerca de la influencia de factores ambientales o secuencias sucesionales (Kent \& Coker 1992). Se utilizó la clasificación de heterogeneidad ambiental de Coria (2014) para determinar el nivel de contraste (bajo: $\mathrm{LG}<3$, medio: $\mathrm{LG}=3-4$, alto: $L G>4$ ), quién tomó como referencia el rango de $\mathrm{LG}=3-4$ que corresponde a una zona intermedia entre la respuesta lineal y unimodal de las variables (Lepš \& Šmilauer 2003). Los análisis de la varianza se realizaron con el software INFOSTAT versión 2013 (Di Rienzo et al. 2013) y el DCA con el software CANOCO 4.56. (Ter Braak \& Šmilauer 2002).

Tabla 1. Abundancia relativa promedio de aves (individuos $/ 10 \mathrm{~min}$ ) en tres tipos de bosques del Chaco Occidental, en épocas reproductiva y no reproductiva (bosque testigo [n=7], RBI antiguo [n=5] y RBI reciente [n=7]). Ver descripción de tratamientos en leyenda de Figura 1. Patagioenas sp. incluye Patagioenas maculosa y P. picazuro. Estatus de Residencia: ver definiciones de categorías en Codesido \& Bilenca (2004).

Table 1. Mean relative abundance (individuals/10 min) of birds in three types of forests in the Western Chaco in reproductive and non-reproductive seasons (untreated forest [ $n=7]$, old RBI [ $n=5]$ and new RBI [ $n=7]$ ). See description of treatments in the legend of Figure 1. Patagioenas sp. includes Patagioenas maculosa and P. picazuro. Residency Status: see definitions of categories in Codesido \& Bilenca (2004).

\begin{tabular}{|c|c|c|c|c|c|c|c|c|}
\hline \multirow[b]{2}{*}{ Especie } & \multirow[b]{2}{*}{$\begin{array}{l}\text { Estatus de } \\
\text { residencia }\end{array}$} & \multirow[b]{2}{*}{ Gremio } & \multicolumn{3}{|c|}{$\begin{array}{l}\text { Época no } \\
\text { reproductiva }\end{array}$} & \multicolumn{3}{|c|}{ Época reproductiva } \\
\hline & & & $\begin{array}{r}\text { re } \\
\text { Bosque } \\
\text { testigo }\end{array}$ & $\begin{array}{l}\text { eproducti } \\
\text { RBI } \\
\text { antiguo }\end{array}$ & $\begin{array}{l}\text { RBI } \\
\text { reciente }\end{array}$ & $\begin{array}{l}\text { Bosque } \\
\text { testigo }\end{array}$ & $\begin{array}{c}\text { RBI } \\
\text { antiguo }\end{array}$ & $\begin{array}{c}\text { RBI } \\
\text { reciente }\end{array}$ \\
\hline Falco femoralis & Residente & Carnívoras insectívoras & & & & & & 0.1 \\
\hline Spiziapteryx circumcincta & Residente & Carnívoras insectívoras & & 0.2 & & & & \\
\hline Elaenia parvirostris & $\begin{array}{l}\text { Migrante } \\
\text { estival }\end{array}$ & Frugívoras & & & & 0.3 & 0.8 & 0.1 \\
\hline Turdus amaurochalinus & $\begin{array}{l}\text { neotropical } \\
\text { Migrante } \\
\text { estival } \\
\text { neotropical }\end{array}$ & Frugívoras & & & & 0.3 & 0.4 & 0.6 \\
\hline Amazona aestiva & Residente & Granívoras arbóreas & & & 0.6 & & & \\
\hline Thectocercus acuticaudatus & Residente & Granívoras arbóreas & 6.6 & 8.0 & 3.0 & & & \\
\hline Myiopsitta monachus & Residente & Granívoras arbóreas & 3.3 & 1.2 & 2.6 & 1.1 & 0.2 & 0.4 \\
\hline Saltator aurantiirostris & Residente & Granívoras arbóreas & 0.4 & 0.8 & 0.1 & 2.3 & 2.2 & 0.9 \\
\hline Leptotila verreauxi & Residente & Granívoras terrestres & & & 0.4 & & 0.8 & \\
\hline Patagioenas sp. & Residente & Granívoras terrestres & 3.7 & 3.8 & 6.6 & 1.0 & 1.4 & 4.3 \\
\hline Columbina picui & Residente & Granívoras terrestres & & & & & 0.2 & 0.3 \\
\hline Poospiza melanoleuca & Residente & Granívoras terrestres & 0.1 & 0.2 & & 0.1 & 0.4 & \\
\hline Saltatricula multicolor & Residente & Granívoras terrestres & & & & & 1.2 & 0.1 \\
\hline Zenaida auriculata & Residente & Granívoras terrestres & 4.6 & 1.8 & 3.1 & 4.7 & 0.6 & 4.4 \\
\hline Zonotrichia capensis & Residente & Granívoras terrestres & 0.3 & 1.0 & 1.9 & 0.4 & 3.4 & 2.9 \\
\hline Milvago chimango & Residente & Insectívoras carroñeras & 0.3 & & 2.1 & & & 1.7 \\
\hline $\begin{array}{l}\text { Campylorhamphus } \\
\text { trochilirostris }\end{array}$ & Residente & Insectívoras de corteza & 0.4 & 0.8 & 0.1 & 0.1 & 0.6 & 0.1 \\
\hline Colaptes melanochloros & Residente & Insectívoras de corteza & 0.6 & 0.4 & 1.7 & 0.7 & 1.2 & 0.9 \\
\hline Drymornis bridgesii & Residente & Insectívoras de corteza & 0.4 & 0.4 & 0.1 & 0.6 & 0.2 & 0.3 \\
\hline $\begin{array}{l}\text { Lepidocolaptes } \\
\text { angustirostris }\end{array}$ & Residente & Insectívoras de corteza & 0.6 & 1.0 & 2.0 & 1.6 & 2.4 & 3.6 \\
\hline Melanerpes cactorum & Residente & Insectívoras de corteza & 0.1 & & & & 0.6 & 0.1 \\
\hline Veniliornis mixtus & Residente & Insectívoras de corteza & 0.1 & 0.2 & 0.7 & 0.1 & & 1.0 \\
\hline Xiphocolaptes major & Residente & Insectívoras de corteza & & 0.2 & & 0.3 & 0.2 & 0.1 \\
\hline Asthenes baeri & Residente & Insectívoras de follaje & 0.4 & 0.6 & 0.3 & 0.6 & 0.6 & 1.1 \\
\hline Cranioleuca pyrrhophia & Residente & Insectívoras de follaje & 0.4 & & 0.4 & 1.1 & 1.6 & \\
\hline Cyclarhis gujanensis & Residente & Insectívoras de follaje & & & & 0.3 & 0.2 & \\
\hline
\end{tabular}


Tabla 1. Continuación.

Table 1. Continuation.

\begin{tabular}{|c|c|c|c|c|c|c|c|c|}
\hline \multirow[b]{2}{*}{ Especie } & \multirow[b]{2}{*}{$\begin{array}{l}\text { Estatus de } \\
\text { residencia }\end{array}$} & \multirow[b]{2}{*}{ Gremio } & \multicolumn{3}{|c|}{$\begin{array}{l}\text { Época no } \\
\text { reproductiva }\end{array}$} & \multicolumn{3}{|c|}{ Época reproductiva } \\
\hline & & & $\begin{array}{l}\text { Bosque } \\
\text { testigo }\end{array}$ & $\begin{array}{c}\text { RBI } \\
\text { antiguo }\end{array}$ & $\begin{array}{c}\mathrm{RBI} \\
\text { reciente }\end{array}$ & $\begin{array}{l}\text { Bosque } \\
\text { testigo }\end{array}$ & $\begin{array}{c}\text { RBI } \\
\text { antiguo }\end{array}$ & $\begin{array}{c}\mathrm{RBI} \\
\text { reciente }\end{array}$ \\
\hline Setophaga pitiayumi & Residente & Insectívoras de follaje & & & & 0.4 & 0.8 & 0.7 \\
\hline Piranga ava & Residente & Insectívoras de follaje & 0.6 & 0.4 & & & 0.4 & \\
\hline Polioptila dumicola & Residente & Insectívoras de follaje & 0.7 & 1.4 & 0.9 & 1.6 & 1.4 & 0.7 \\
\hline Pseudoseisura lophotes & Residente & Insectívoras de follaje & 0.6 & 0.4 & 0.1 & 0.7 & 1.2 & 0.9 \\
\hline Stigmatura budytoides & Residente & Insectívoras de follaje & 1.7 & 2.6 & 3.3 & 1.6 & 1.8 & 2.3 \\
\hline Synallaxis albescens & Residente & Insectívoras de follaje & & & & & 0.8 & \\
\hline Tapera naevia & $\begin{array}{l}\text { Migrante } \\
\text { estival } \\
\text { neotropical }\end{array}$ & Insectívoras de follaje & & & & & & 0.1 \\
\hline Troglodytes aedon & Residente & Insectívoras de follaje & & 0.2 & 0.4 & & & 0.1 \\
\hline Tarphonomus certhioides & Residente & Insectívoras de follaje & 0.6 & 0.2 & 0.1 & 0.9 & 1.0 & 0.1 \\
\hline Vireo olivaceus & $\begin{array}{l}\text { Migrante } \\
\text { estival }\end{array}$ & Insectívoras de follaje & & & & 2.3 & 1.8 & 2.6 \\
\hline Camptostoma obsoletum & $\begin{array}{l}\text { neotropical } \\
\text { Migrante } \\
\text { estival }\end{array}$ & Insectívoras de vuelo & & & & 0.3 & 0.8 & 1.6 \\
\hline Euscarthmus meloryphus & $\begin{array}{l}\text { neotropical } \\
\text { Migrante } \\
\text { estival }\end{array}$ & Insectívoras de vuelo & & & & 1.3 & 0.8 & \\
\hline $\begin{array}{l}\text { Empidonomus } \\
\text { aurantioatrocristatus }\end{array}$ & $\begin{array}{l}\text { neotropical } \\
\text { Migrante } \\
\text { estival } \\
\text { neotronical }\end{array}$ & Insectívoras de vuelo & & & & 0.1 & 1.0 & 0.1 \\
\hline $\begin{array}{l}\text { Hemitriccus } \\
\text { margaritaceiventer }\end{array}$ & Residente & Insectívoras de vuelo & & & & & & 0.3 \\
\hline Knipolegus aterrimus & Accidental & Insectívoras de vuelo & & & 0.1 & & & \\
\hline Knipolegus striaticeps & Residente & Insectívoras de vuelo & & 0.4 & 0.1 & 0.1 & & 0.1 \\
\hline Myiarchus swainsoni & $\begin{array}{l}\text { Migrante } \\
\text { estival }\end{array}$ & Insectívoras de vuelo & & & & 1.3 & 0.6 & 0.7 \\
\hline Myiarchus tyrannulus & $\begin{array}{l}\text { neotropical } \\
\text { Migrante } \\
\text { estival }\end{array}$ & Insectívoras de vuelo & & & & 0.3 & 0.4 & 0.4 \\
\hline $\begin{array}{l}\text { Pachyramphus } \\
\text { polychopterus }\end{array}$ & $\begin{array}{l}\text { neotropical } \\
\text { Migrante } \\
\text { estival }\end{array}$ & Insectívoras de vuelo & & & & 0.4 & & 0.1 \\
\hline Sublegatus modestus & $\begin{array}{l}\text { neotropical } \\
\text { Migrante } \\
\text { estival } \\
\text { neotropical }\end{array}$ & Insectívoras de vuelo & & & & 0.3 & 0.2 & 0.1 \\
\hline Suiriri suiriri & Residente & Insectívoras de vuelo & 2.4 & 2.4 & 2.9 & 2.1 & 2.0 & 2.3 \\
\hline Agelaioides badius & Residente & Insectívoras terrestres & & & & & 0.4 & 0.1 \\
\hline Furnarius rufus & Residente & Insectívoras terrestres & 0.4 & & & 0.3 & 0.6 & \\
\hline Molothrus rufoaxillaris & Dudosa & Insectívoras terrestres & 0.1 & 0.6 & & & 0.4 & \\
\hline Molothrus bonariensis & Dudosa & Insectívoras terrestres & & 0.2 & 0.1 & 0.3 & & \\
\hline Rhinocrypta lanceolata & Residente & Insectívoras terrestres & 0.6 & 0.6 & 0.3 & 0.4 & 0.4 & 0.1 \\
\hline Chlorostilbon lucidus & $\begin{array}{l}\text { Migrante } \\
\text { estival }\end{array}$ & Nectarívoras & & & & & & 0.4 \\
\hline Heliomaster furcifer & $\begin{array}{l}\text { neotropical } \\
\text { Migrante } \\
\text { estival } \\
\text { neotropical }\end{array}$ & Nectarívoras & & & & 0.1 & 0.2 & 1.0 \\
\hline Chunga burmeisteri & Residente & Omnívoras & 1.0 & 0.8 & 0.9 & 0.3 & 1.0 & 0.9 \\
\hline Crypturellus tataupa & Residente & Omnívoras & & & & 0.4 & 0.8 & 0.1 \\
\hline Nothoprocta cinerascens & Residente & Omnívoras & & & & & 0.4 & 0.9 \\
\hline Ortalis canicollis & Residente & Omnívoras & & 0.6 & 0.6 & & 1.0 & 3.0 \\
\hline
\end{tabular}

\section{RESUlTADOS}

En total se registraron 58 especies de aves, 35 en la época no reproductiva y 54 en la reproductiva (Tabla 1). Fueron registrados en total 1348 individuos, 437 en el bosque testigo, 354 en el RBI antiguo y 557 en el
RBI reciente. Discriminados por época, 718 correspondieron a la estación reproductiva y 630 a la no reproductiva. Las curvas de acumulación de especies mostraron una tendencia a la estabilización, en especial en la época no reproductiva, lo que indica que 
a. Época no Reproductiva

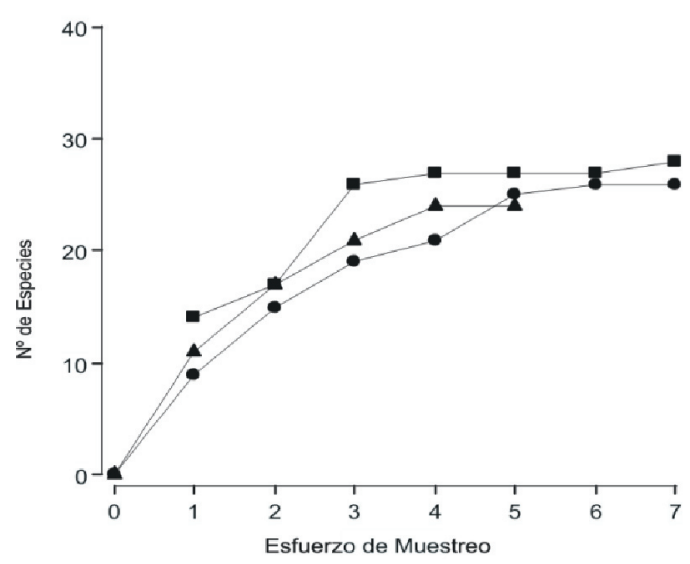

b. Época Reproductiva

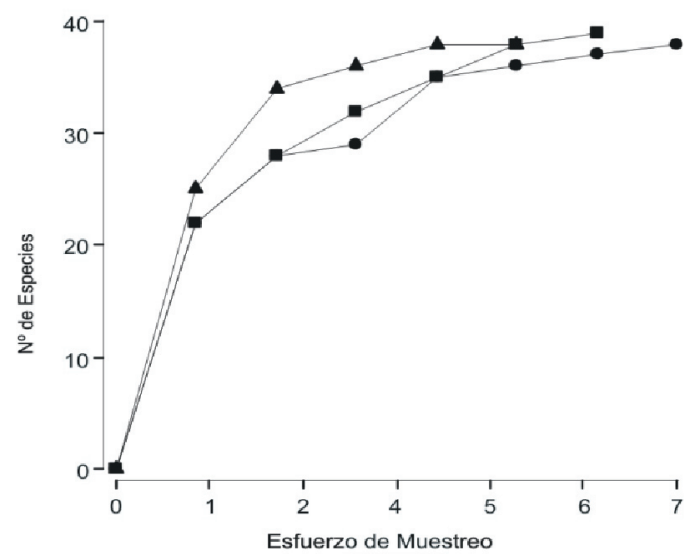

Figura 1. Curvas de acumulación de especies de aves en épocas no reproductiva (a) y reproductiva (b). Los hábitats comparados son tres tipos de bosques del Chaco Occidental: i) bosque testigo (círculos): bosque con ganadería sin aprovechamiento forestal en tres décadas, ii) RBI antiguo (triángulos): bosque de 32 ha tratado con RBI, con siembra de la pastura exótica Megathyrsus maximus cv gatton panic, con pastoreo ganadero y 30\% del área basal de árboles extraída, de siete años de antigüedad, y iii) RBI reciente (cuadrados): bosque de 100 ha tratado con RBI, sin siembra de pasturas exóticas, con pastoreo ganadero, con $30 \%$ del área basal apeada pero extraída sólo el $8.2 \%$, de un año de antigüedad.

Figure 1. Cummulative bird species curves in nonreproductive (a) and reproductive (b) seasons. Environments under comparison are: i) untreated forest (circles): forest with livestock and without logging in three decades, ii) old RBI (triangles): 32 ha forest treated with RBI and exotic pastures of Megathyrsus maximus cv gatton panic, with livestock grazing and $30 \%$ of basal area of trees removed, seven years old, and iii) new RBI (squares): 100 ha forest treated with RBI, without exotic pastures, with livestock grazing, with $30 \%$ of the basal area of trees felled but extracted only $8.2 \%$, a year old.

el número de especies estuvo bien estimado, pudiendo quedar fuera del inventario especies muy escasas (Martella et al. 2012) (Figura 1).

\section{Contrastes de hábitat y diversidades alfa y beta}

El contraste de hábitat entre los tres tipos de bosque fue bajo $(\mathrm{LG}<3)$. La diversidad alfa de aves no disminuyó en ambos bosques con RBI (incluso fue un poco mayor en RBI reciente), además fue mayor en la época reproductiva (Figura 2a). Se determinó un mayor contraste de hábitat entre bosque testigo-RBI reciente que entre bosque testigoRBI antiguo, reflejado en la segregación más acentuada entre los primeros y en el menor valor de LG del eje 2 a lo largo del cual se distribuyen los puntos de bosque testigo-RBI antiguo (Figura 3). La diversidad beta de aves en todos los casos fue baja y mayor entre los bosques más contrastados: bosque testigo-RBI antiguo, época reproductiva: $\mathrm{Jd}=0.29$, época no reproductiva: $\mathrm{Jd}=0.31$; bosque testigo-RBI reciente, época reproductiva: $\mathrm{Jd}=0.36$, época no reproductiva: $\mathrm{Jd}=0.36$.

\section{Gremios tróficos}

En la época no reproductiva se registraron nueve gremios tróficos, mientras que en la reproductiva se adicionaron otros dos (nectarívoras y frugívoras), dando un total de 11 (Tabla 1). Tres gremios presentaron diferencias asociadas a los tratamientos: las insectívoras carroñeras fueron más abundantes en RBI reciente (mayor abundancia de Milvago chimango), las insectívoras terrestres fueron menos abundantes en RBI reciente (menor riqueza y abundancia de especies en ambas épocas), y las nectarívoras fueron más abundantes en RBI reciente (mayor abundancia de Heliomaster furcifer) (Figura $2 \mathrm{~b}$, c y k; Tabla 1). Dos gremios presentaron diferencias asociadas a los tratamientos y las épocas: las granívoras arbóreas fueron menos abundantes en RBI reciente (menores abundancias de Thectocercus acuticaudatus y Saltator aurantiirostris) y más abundantes en la época no reproductiva (mayores abundancias de Thectocercus acuticaudatus y Myiopsitta monachus), y las insectívoras de corteza fueron más abundantes en RBI reciente (mayores abundancias de Lepidocolaptes angustirostris) y más abundantes en la época reproductiva (Figura 2d y e; Tabla 1). Las omnívoras, insectívoras de vuelo e insectívoras de follaje presentaron diferencias asociadas a la época, siendo más abundantes en la época reproductiva (Figura 2f, g y h). Las granívoras terrestres y carnívoras insectívoras no variaron entre tratamientos y épocas (Figura $2 \mathrm{i}$ y j). Las frugívoras (sólo en época reproductiva) no variaron entre tratamientos (Figura 21). 

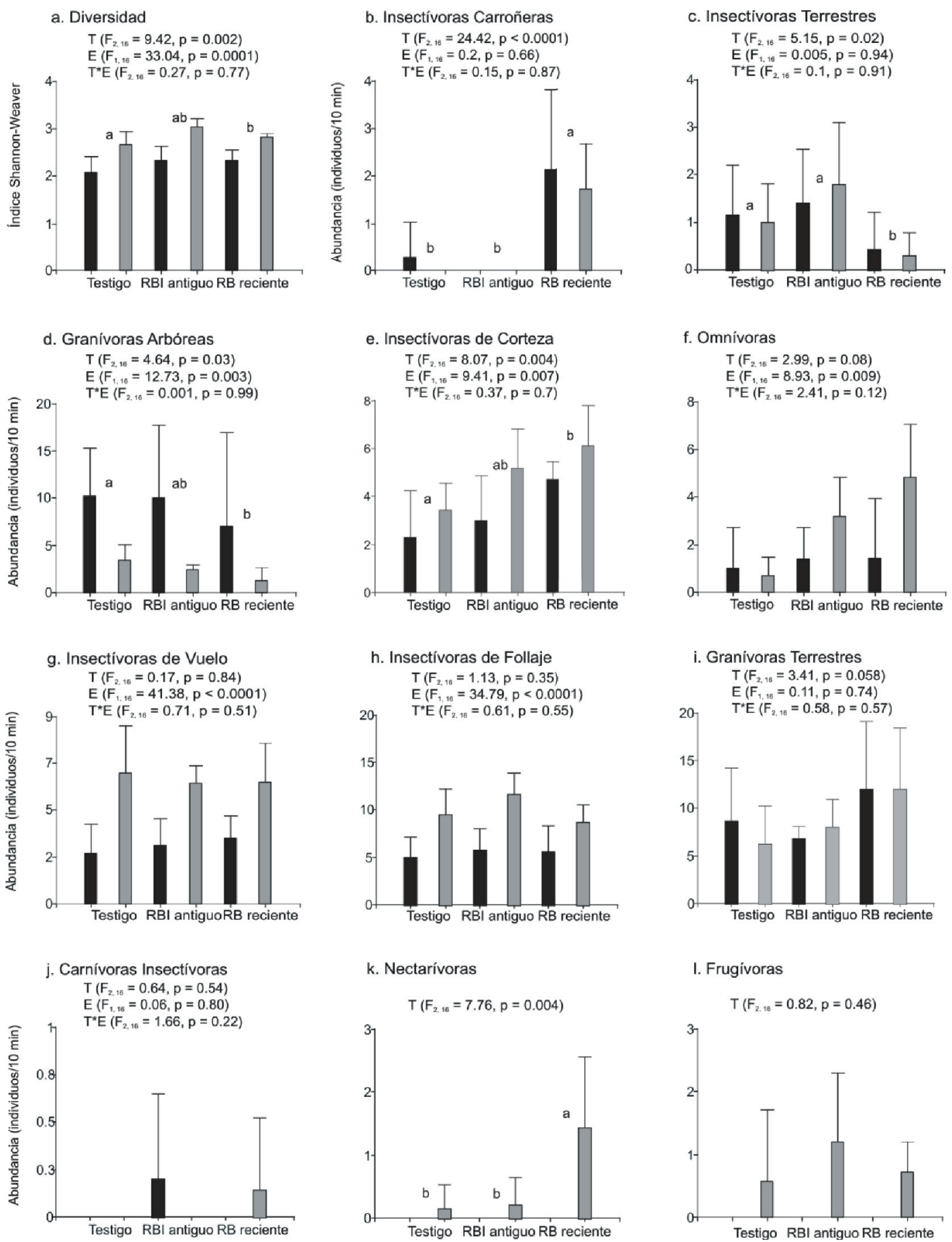

k. Nectarívoras

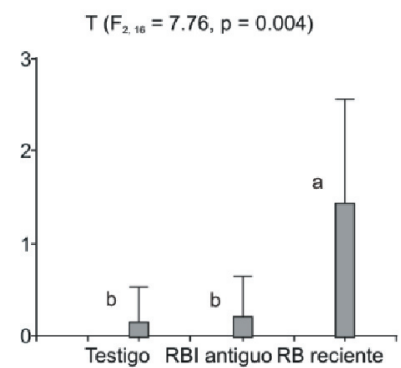

I. Frugivoras

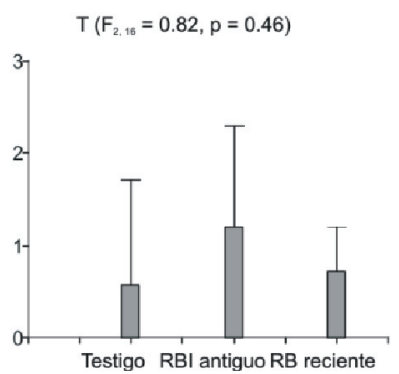

Figura 2. Abundancia de gremios tróficos y diversidad alfa (índice Shannon-Weaver) de aves en tres tipos de bosques del Chaco Occidental (media+desvío estándar; bosque testigo [n=7], RBI antiguo [n=5] y RBI reciente [n=7]). $\mathrm{T}=$ tratamientos (ver descripción en la leyenda de la Figura 1). E=épocas (no reproductiva [barras negras]; reproductiva [barras grises]). Gráficos desde " $a$ " hasta " $j$ " MANOVA de un factor (T) con enfoque de medidas repetidas en el tiempo (E, no reproductiva y reproductiva). Gráficos " $\mathrm{k}$ " y " 1 " ANOVA de un factor (T). Las barras con una letra común indican que los tratamientos no fueron significativamente diferentes (prueba de Hotelling para MANOVA o prueba de Tukey para ANOVA). En todas las pruebas se estableció $\alpha=0.05$.

Figure 2. Abundances of trophic guilds and alpha diversity (Shannon-Weaver index) of birds in three types of forests of Western Chaco (mean+standard deviation; untreated forest [ $n=7]$, old RBI [ $n=5]$ and new RBI [ $n=7]$ ). T=treatment (see description in legend of Figure 1). E=season (no reproductive [black bars]; reproductive [gray bars]). Graphics from " $a$ " to " $j$ " MANOVA of one factor $(T)$ with repeated measures approach in time (E, non-breeding and breeding). Graphics " $k$ " and " 1 " ANOVA of one factor (T). Means with the same letter indicate that the treatments were not significantly different (Hotelling test for MANOVA or Tukey test for ANOVA). $\alpha=0.05$ for all tests. 
Tabla 2. Medias de frecuencias de especies vegetales de tres tipos de bosques del Chaco Occidental (bosque testigo [n=7], RBI antiguo [n=5] y RBI reciente [n=7]). Ver descripción de tratamientos y arreglos de vegetación (arbustal, arbolado) en las leyendas de las Figuras 1 y 4 .

Table 2. Mean frequency of plant species in three types of forest in the Western Chaco (untreated forest [n=7], old RBI $[n=5]$ and new RBI [ $n=7]$ ). See description of treatments and vegetation arrangement (arbustal, arbolado) in legends of Figures 1 and 4.

\begin{tabular}{|c|c|c|c|c|c|c|c|}
\hline & \multirow[b]{2}{*}{ Especies } & \multicolumn{2}{|c|}{ Bosque testigo } & \multicolumn{2}{|c|}{ RBI antiguo } & \multicolumn{2}{|c|}{ RBI reciente } \\
\hline & & Arbustal & Arbolado & Arbustal & Arbolado & Arbustal & Arbolado \\
\hline \multirow[t]{8}{*}{ Árboles } & Aspidosperma quebracho-blanco & 42.9 & 76.2 & 66.7 & 73.3 & 61.9 & 76.2 \\
\hline & Schinopsis lorentzii & 23.8 & 33.3 & 66.7 & 66.7 & 61.9 & 42.9 \\
\hline & Ziziphus mistol & 33.3 & 23.8 & 26.7 & 6.7 & 33.3 & 47.6 \\
\hline & Prosopis nigra & 28.6 & 19.0 & 13.3 & 13.3 & 14.3 & 19.0 \\
\hline & Cercidium praecox & 28.6 & 4.8 & 20.0 & 0.0 & 9.5 & 0.0 \\
\hline & Castela coccinea & 9.5 & 9.5 & 0.0 & 0.0 & 0.0 & 4.8 \\
\hline & Jodina rhombifolia & 4.8 & 0.0 & 0.0 & 0.0 & 0.0 & 4.8 \\
\hline & Opuntia quimilo & 4.8 & 0.0 & 0.0 & 0.0 & 0.0 & 0.0 \\
\hline \multirow[t]{12}{*}{ Arbustos } & Celtis ehrenbergiana & 42.9 & 38.1 & 20.0 & 53.3 & 61.9 & 81.0 \\
\hline & Acacia gilliesii & 28.6 & 28.6 & 46.7 & 53.3 & 38.1 & 28.6 \\
\hline & Larrea divaricata & 0.0 & 0.0 & 0.0 & 0.0 & 23.8 & 9.5 \\
\hline & Moya spinosa & 4.8 & 4.8 & 13.3 & 6.7 & 14.3 & 9.5 \\
\hline & Capparis atamisquea & 52.4 & 61.9 & 40.0 & 46.7 & 4.8 & 9.5 \\
\hline & Condalia microphylla & 0.0 & 4.8 & 0.0 & 0.0 & 4.8 & 4.8 \\
\hline & Lycium tenuispinosum & 9.5 & 0.0 & 0.0 & 6.7 & 4.8 & 0.0 \\
\hline & Schinus sp. & 4.8 & 0.0 & 0.0 & 6.7 & 4.8 & 0.0 \\
\hline & Ximenia americana & 4.8 & 0.0 & 0.0 & 0.0 & 0.0 & 0.0 \\
\hline & Acacia aroma & 4.8 & 0.0 & 13.3 & 0.0 & 0.0 & 0.0 \\
\hline & Justicia xylosteoides & 0.0 & 4.8 & 6.7 & 0.0 & 0.0 & 0.0 \\
\hline & Lippia turbinata & 4.8 & 0.0 & 0.0 & 0.0 & 0.0 & 0.0 \\
\hline \multirow{6}{*}{$\begin{array}{l}\text { Latifoliada } \\
\text { herbáceas }\end{array}$} & Abutilon sp. & 0.0 & 0.0 & 0.0 & 6.7 & 0.0 & 14.3 \\
\hline & Justicia squarrosa & 38.1 & 28.6 & 26.7 & 6.7 & 0.0 & 9.5 \\
\hline & Wissadula densiflora & 14.3 & 28.6 & 6.7 & 13.3 & 0.0 & 4.8 \\
\hline & Latifoliadas indeterminadas & 23.8 & 28.6 & 6.7 & 40.0 & 0.0 & 4.8 \\
\hline & Lantana sp. & 4.8 & 0.0 & 0.0 & 0.0 & 0.0 & 0.0 \\
\hline & Amphilophium carolinae & 0.0 & 0.0 & 0.0 & 6.7 & 0.0 & 0.0 \\
\hline \multirow[t]{5}{*}{ Gramíneas } & Setaria sp. & 0.0 & 9.5 & 0.0 & 6.7 & 0.0 & 4.8 \\
\hline & Gouinia latifolia & 4.8 & 4.8 & 0.0 & 0.0 & 4.8 & 0.0 \\
\hline & Megathyrsus maximus co gatton panic & 0.0 & 0.0 & 26.7 & 66.7 & 0.0 & 0.0 \\
\hline & Setaria lachnea & 0.0 & 0.0 & 0.0 & 6.7 & 0.0 & 0.0 \\
\hline & Trichloris crinita & 14.3 & 4.8 & 13.3 & 0.0 & 0.0 & 0.0 \\
\hline
\end{tabular}

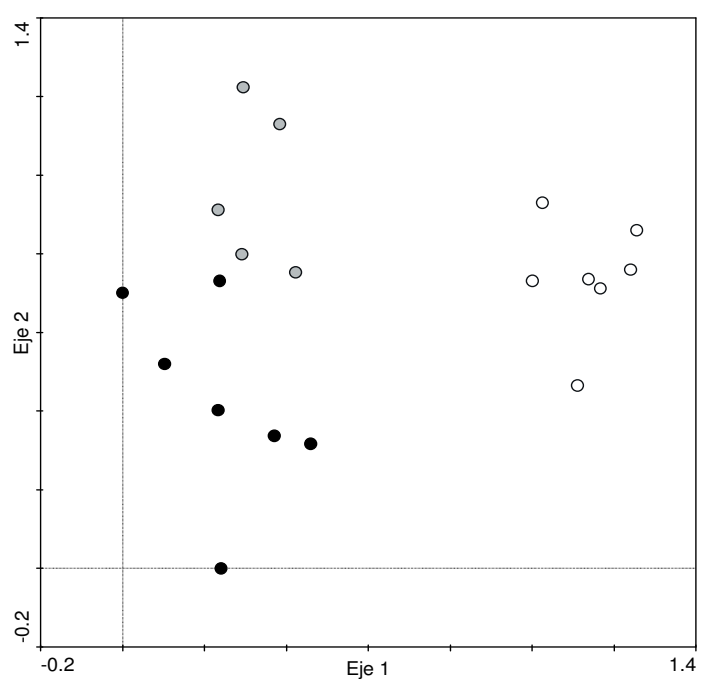

Figura 3. Diagrama de ordenamiento DCA de unidades de muestreo, de acuerdo con las variables de vegetación medidas en tres tipos de bosques del Chaco Occidental: bosque testigo (círculos oscuros), RBI antiguo (grises) y RBI reciente (blancos). Ver descripción de tratamientos en leyenda de Figura 1. Eje 1: LG=1.255; eje 2: $L G=1.224$.

Figure 3. Ordination diagram DCA of sampling units according to vegetation variables measured in three forest types of the Western Chaco: untreated forest (dark circles), old RBI (gray) and new RBI (white). See description of treatments in the legend of Figure 1. Axis 1: $L G=1.255$; axis 2 : $L G=1.224$. 

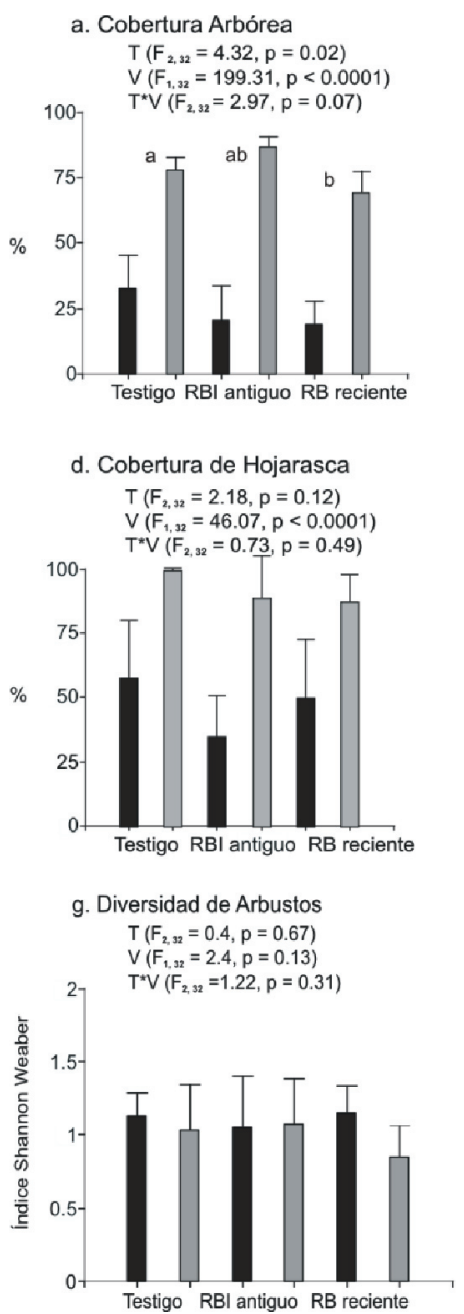
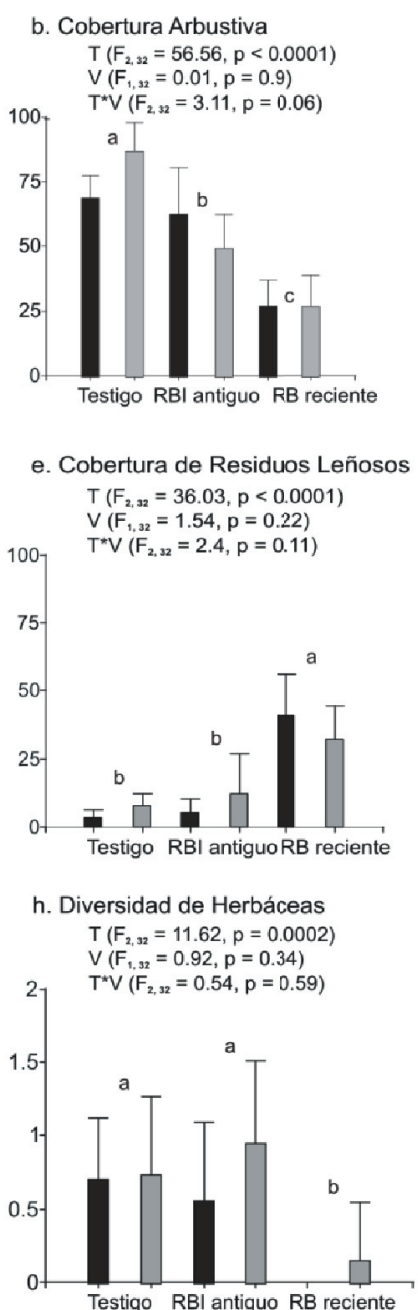

c. Cobertura Herbácea

$T\left(F_{2,32}=3.43, p=0.04\right)$

$V\left(F_{1,32}=0.1, p=0.76\right)$

$100-\quad T^{*} V\left(F_{2,32}=1.47, p=0.24\right)$

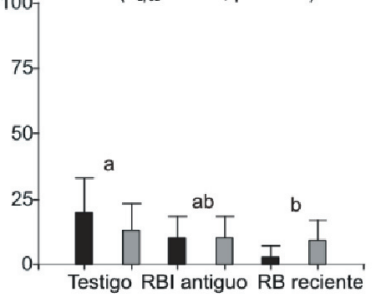

f. Diversidad de Árboles

$T\left(F_{2,32}=2.4, p=0.11\right)$

$V\left(F_{1,32}=1.46, p=0.24\right)$

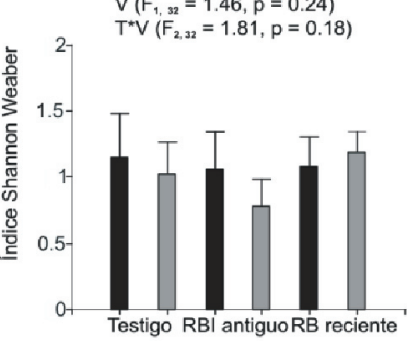

Figura 4. Variables de vegetación medidas en tres tipos de bosques del Chaco Occidental (media+desvío estándar; bosque testigo [n=7], RBI antiguo [n=5] y RBI reciente [n=7]). T=tratamientos (ver descripción en la leyenda de la Figura 1). V=arreglos de vegetación (arreglo arbustal [barras negras]: sectores del bosque con cobertura arbustiva predominante; arreglo arbolado [barras grises]: sectores del bosque con cobertura arbórea predominante). ANOVA de dos factores ( $\mathrm{T}$ y V). Medias con una letra común indican que los tratamientos no fueron significativamente diferentes (prueba de Tukey). En todas las pruebas se estableció $\alpha=0.05$.

Figure 4. Vegetation variables measured in three types of forests of Western Chaco (mean+standard deviation; untreated forest [n=7], old RBI [n=5] and new RBI [n=7]). T=treatment (see description in legend of Figure 1). V=vegetation arrangement (arreglo arbustal [black bars]: sectors of the forest with predominant shrub cover; arreglo arbolado [gray bars]: sectors of the forest with dominant tree cover). Two-way ANOVA (T and V). Means with the same letter indicate that the treatments were not significantly different (Tukey Test). $\alpha=0.05$ in all tests.

\section{Vegetación}

La cobertura arbórea fue 20.6 \% menor en RBI reciente y mayor en el arreglo arbolado (Figura 4a) (prueba de Tukey para el factor arreglo de vegetación, $P<0.05)$, mientras que la cobertura arbustiva fue directamente proporcional con la edad del tratamiento (bosque testigo [77.6\%] $>$ RBI antiguo [56\%]>RBI reciente [26.7\%]) (Figura 4b). La cobertura herbácea fue $62.8 \%$ menor en el RBI reciente (Figura $4 \mathrm{c}$ ), la de hojarasca fue la misma entre tratamientos pero mayor en el arreglo arbolado (Figura 4d; prueba de Tukey para el factor arreglo de vegetación, $P<0.05)$, y la de residuos leñosos se incrementó en $563.1 \%$ en RBI reciente (Figura 4e). En la Tabla 2 se presentan las frecuencias de especies a partir de las cuales se obtuvieron los índices de diversidad. Las diversidades alfa de árboles y arbustos fueron las mismas entre tratamientos, pero la de herbáceas disminuyó en el RBI reciente (Figura $4 \mathrm{f}, \mathrm{g}$ y h).

\section{DisCUSIÓN}

\section{Contraste de hábitats y diversidades alfa y beta}

En la Región Chaqueña, la intensificación de la producción ganadera modifica los hábitats y 
ejerce efectos adversos en las diversidades de aves de bosques (Mastrangelo \& Gavin 2012; Macchi et al. 2013). Nuestros resultados son consistentes con los de estudios previos. En un bosque tratado con rolado selectivo de baja intensidad (RBI) de dos años de edad no hubo cambios significativos en la diversidad alfa de aves (Coria et al. 2012), al igual que en otros SSPs de baja intensidad (Mastrangelo \& Gavin 2012). En un gradiente productivo ganadero del Chaco Occidental, las similitudes entre los ensambles de aves decrecieron de forma no lineal con el incremento de la intensificación de la producción (i.e., bosques $<$ sistemas de muy baja intensidad $<$ SSPs de baja intensidad $<$ SSPs de intensidades intermedias $<$ SPs de alta intensidad) (Mastrangelo \& Gavin 2012). En las Sierras de Guasayán (Chaco Serrano) se evaluaron aves en tres hábitats con bajos contrastes $(\mathrm{LG}<3)$ (i.e., ladera húmeda, ladera seca y piedemonte oriental) y se encontró que los hábitats menos contrastados (i.e., laderas húmeda y seca) tuvieron la menor diversidad beta (Coria 2014). En un estudio previo los bosques del área de estudio presentaron mayor diversidad alfa de aves en la época reproductiva frente a la no reproductiva (Codesido \& Bilenca 2004). Nuestros resultados sugieren que respecto al bosque no perturbado usado como referencia: i) los dos bosques tratados con RBI tuvieron bajo contraste, ii) la diversidad alfa de aves no disminuyó a causa del RBI (incluso fue un poco mayor en el tratamiento reciente), iii) la variación estacional de la abundancia y riqueza de aves (mayores en la época reproductiva) se mantuvieron en los tres bosques estudiados, y iv) en ambas épocas los tratamientos con RBI tuvieron baja diversidad beta de aves. Estos resultados son consistentes con nuestras hipótesis (H1 y H3) acerca del bajo impacto del RBI sobre la vegetación y las aves. Además, el tratamiento RBI antiguo mostró los niveles más bajos de contraste y diversidad beta respecto al bosque testigo, lo cual da soporte a nuestras hipótesis acerca de la recuperación de la vegetación afectada por el RBI con el tiempo y su consecuente efecto sobre la comunidad de aves ( $\mathrm{H} 2$ y H3).

El RBI genera bosques parcialmente abiertos que retienen gran parte de su estructura y composición florística original y que tienden a cerrarse con el tiempo dada la recuperación de la cobertura arbustiva (Kunst et al. 2008, 2012; Albanesi et al. 2013). En nuestro estudio: i) el RBI antiguo mostró pocos cambios asociados al tratamiento, sólo tuvo lugar una reducción de la cobertura arbustiva $(27.8 \%)$ y una alta frecuencia de la pastura implantada Gatton panic (47\%), y ii) el RBI reciente mostró mayores cambios asociados al tratamiento, con reducción de coberturas arbórea (20.6\%), arbustiva $(65.6 \%)$, herbácea $(62.8 \%)$ y de la diversidad de herbáceas e incremento de la cobertura de residuos leñosos (563.1\%), aunque mantuvo sin variación las diversidades de árboles y arbustos y la cobertura de hojarasca. Estos cambios fueron evidentes también en el mayor contraste encontrado entre el bosque testigo y el RBI reciente en el ordenamiento multivariado (DCA). A pesar de estos cambios significativos en algunas variables, en general la heterogeneidad ambiental detectada fue baja, sin un recambio importante de vegetación entre los hábitats; de hecho, el ecosistema de bosque se mantuvo en los RBI.

Las matrices productivas de bajo contraste favorecen el funcionamiento de los ecosistemas, a través del suministro de hábitats para las especies, mayor conectividad y disminución de efectos de borde (Fischer et al. 2006). Los bajos contrastes de hábitats que encontramos sugieren que las matrices con RBI presentan potencial para utilizarse como áreas "buffer" alrededor de vegetación de alto valor de conservación para las aves (e.g., áreas protegidas, relictos de vegetación y corredores biológicos). Estoes consistenteconMastrangelo \& Gavin (2012, 2014) y Mastrangelo (2014), quienes ya habían mostrado que los SSPs de baja intensidad de la Región Chaqueña tienen elevado valor de conservación para las aves. En este sentido, las matrices de mayor contraste (SSPs de intensidad intermedia, SPs de alta intensidad y cultivos) seguramente son menos adecuadas.

Nuestros resultados sugieren que los tratamientos con RBI de mayor edad mantienen las composiciones de especies de aves más similares a los bosques de referencia, por lo cual tienen un valor extra de conservación. Sin embargo, desde la perspectiva productiva los tratamientos de mayor edad plantean los mismos inconvenientes que motivaron los disturbios (i.e., baja accesibilidad y producción de forraje), por lo cual la vegetación debe ser re-rolada con una frecuencia no menor a cinco años para mantener índices productivos satisfactorios (Albanesi et al. 2013). El Manual del RBI recomienda utilizar un esquema de rotaciones donde la superficie total de manejo se divida en 20 lotes y donde coexistan lotes tratados con RBI de todas las edades (consultar fundamentos en Kunst et al. 2008). Nuestro estudio sugiere que este esquema de manejo resultaría apropiado para las aves por dos 
motivos: i) los tratamientos de mayor edad conservan mejor la composición de especies de aves de bosque y (ii) el mayor recambio de especies de aves que ocurre en los tratamientos de menor edad significa un incremento del número de especies para el mosaico ambiental generado. Un mosaico de lotes con RBI de distintas edades con superficies mayores a 100 ha cada uno (umbral surgido del tamaño de RBI reciente en nuestro estudio) produciría el efecto mencionado. Una limitación para hacer estas inferencias es que nuestro estudio tuvo un pequeño tamaño de muestra determinado por los tamaños de los tratamientos evaluados y que no consideramos efectos de borde y de paisaje. Si bien en matrices ganaderas del Chaco Occidental la estructura de la vegetación a escala de lote es el factor de mayor peso en la determinación de la diversidad de aves, los efectos de borde y paisaje ejercen cierta influencia (Mastrangelo \& Gavin 2014).

\section{Gremios tróficos}

Los cambios en la estructura y composición de la vegetación producen respuestas en los gremios tróficos de aves porque influyen en el acceso y la cantidad de recursos disponibles en cada microhábitat (i.e., tipo de alimento consumido, lugar de alimentación, requerimientos para la nidificación y afinidad por tipos de coberturas) (Lopez de Casenave et al. 1998; Lopez de Casenave 2001; Milesi et al. 2002), aunque los hábitos comportamentales de ciertas especies pueden hacerlas menos sensibles a las variaciones de los hábitats (Cueto 1996). Nuestros resultados indican que: i) todas las variaciones en las abundancias gremiales asociadas al RBI se produjeron en el tratamiento de mayor contraste (RBI reciente), lo cual es consistente con la predicción de la hipótesis de contraste (H3), y ii) las variaciones estacionales en las abundancias gremiales (mayores en la época reproductiva) fueron evidentes en los tres tipos de bosque.

En RBI reciente, los incrementos de insectívoras carroñeras, insectívoras de corteza y nectarívoras estuvieron asociados a incrementos de Milvago Chimango, Lepidocolaptes angustirostris y Heliomaster furcifer, respectivamente. La apertura de la vegetación leñosa posiblemente favoreció a estas especies. La primera es afín a hábitats abiertos y se adapta bien a hábitats antropizados (Biondi et al. 2005; Di Giacomo 2005; RD Coria, observación personal). La segunda se desplaza entre árboles para alimentarse (Codesido et al. 2009) y es frecuente en áreas despejadas y antropizadas (Di Giacomo 2005; RD Coria, observación personal). La tercera estuvo presente sólo en la época reproductiva, y su incremento puede deberse a que nidifica en árboles grandes y aislados (Di Giacomo 2005), requerimientos que se cumplen en el tratamiento reciente.

Por el contrario, las insectívoras terrestres y granívoras arbóreas evidenciaron menores abundancias en el hábitat más disímil al bosque testigo (RBI reciente). Cuatro de las especies que componen el primer gremio (i.e., Furnarius rufus, Agelaioides badius, Molothrus rufoaxillaris y $M$. bonariensis) son generalistas de hábitats, mientras que una (i.e., Rhinocrypta lanceolata) es afín a coberturas leñosas cerradas (Codesido et al. 2009; RD Coria, observación personal). Las reducciones de las coberturas vegetales posiblemente hayan impactado de forma negativa en la abundancia de insectos en el suelo, y producido el decrecimiento observado en este gremio. Además, R. lanceolata nidifica a baja altura en el sotobosque (Fraga \& Narosky 1985) y pudo ser afectada por una menor calidad del hábitat para nidificar (Codesido et al. 2009). Las granívoras arbóreas decrecieron en particular por las menores abundancias de Thectocercus acuticaudatus y Saltator aurantiirostris. Estas especies se alimentan sobre las leñosas ( $S$. aurantiirostris lo hace además en el suelo) (Di Giacomo 2005) y es posible que la reducción de la cobertura leñosa haya impactado negativamente en los alimentos disponibles para estas especies (Codesido et al. 2009).

En general, los gremios fueron más abundantes en la época reproductiva o no presentaron variación estacional. Los incrementos en la época reproductiva estuvieron asociados al arribo de especies migrantes estivales neotropicales y la mayor abundancia de aves en general, patrones consistentes con lo previamente informado para los bosques no perturbados del área de estudio (Codesido \& Bilenca 2004). Sólo las granívoras arbóreas presentaron mayor abundancia en la época no reproductiva, debido a incrementos de Thectocercus acuticaudatus y Myiopsitta monachus. Estos psitácidos generalistas presentan gran movilidad en función de los alimentos disponibles en los distintos parches o matrices del paisaje (Fernández et al. 1997; Canavelli 2010). En esta época seca posiblemente el bosque ofreció más alimentos en relación a las áreas circundantes (e.g., cultivos) (RD Coria, observación personal). Otro factor de 
importancia pudo haber sido la presencia de cactáceas en el bosque, ya que estas aves son consumidoras asiduas de estas plantas como fuente alternativa de agua (Bucher 1980).

La Región Chaqueña se caracteriza por su gran irregularidad en sus precipitaciones, tanto espacial como temporal, que producen variaciones en las coberturas vegetales y la disponibilidad de alimentos y agua libre para la fauna (Bucher 1980). Este estudio se realizó en un momento en que el área de estudio atravesaba una sequía de dos años (precipitación total 2012 $/ 2013=599 \mathrm{~mm}$ ) (INTA EEA Santiago del Estero 2014), lo que puede explicar la menor riqueza de aves observada respecto a un estudio previo (Coria et al. 2012) realizado en la misma área en una época de precipitaciones mayores $(2008 / 2009=868$ mm) (INTA EEA Santiago del Estero 2014). La riqueza en nuestro estudio fue $15.6 \%$ menor en la época reproductiva y $28.5 \%$ menor en la no reproductiva.

\section{Conclusiones e implicancias para el manejo}

El rolado selectivo de baja intensidad (RBI) se difunde con fuerza en ámbitos gubernamentales y productivos (observación personal de los autores) dadas sus ventajas para la producción ganadera y forestal en un marco de sustentabilidad de los recursos suelo y vegetación (Kunst et al. 2008; Albanesi et al. 2013). Este estudio sugiere que los bosques tratados con RBI mantuvieron la calidad del hábitat para las aves tanto en la época reproductiva como no reproductiva y los grados de contraste de hábitats respondieron a la edad de los tratamientos (a mayor edad, menor contraste). En los bosques con RBI: i) no hubo disminuciones de la diversidad alfa asociadas a los tratamientos, ii) la diversidad beta permaneció baja en ambas épocas, en particular en el tratamiento de mayor edad, y iii) las estructuras de gremios tróficos de aves presentaron algunas modificaciones asociadas al RBI sólo en el tratamiento de menor edad.

Las potenciales implicancias de manejo derivadas de este estudio son: i) el RBI puede satisfacer objetivos de conservación de aves y producción sustentable dados los bajos contrastes de hábitats, lo que sugiere una aptitud para generar matrices productivas poco transformadas que funcionen como áreas buffer alrededor de áreas de alto valor de conservación para las aves (e.g., áreas protegidas, relictos de vegetación y corredores biológicos), y ii) la coexistencia de lotes (>100 ha) con RBI de diferentes edades puede promover la mantención de las especies de bosque y la incorporación de nuevas especies al mosaico ambiental. Se sugiere que nuevos estudios aborden específicamente estas implicancias de manejo en diversos contextos de paisajes y con superficies de bosques más extensos para lograr recomendaciones de manejo genéricas vinculadas al RBI y la conservación de las aves de bosques del Chaco Occidental.

Agradecimientos: Expresamos nuestro agradecimiento al Sr. V. Navarrete por su ayuda en los muestreos de campo y a los revisores anónimos que colaboraron desinteresadamente en la mejora de este trabajo. Esta investigación fue financiada por el Instituto Nacional de Tecnología Agropecuaria (Argentina), en el marco de los Proyectos Específicos N $N^{\circ} 1128053$ y 1126074, año 2013. Los dos primeros autores contribuyeron en partes iguales al desarrollo de este trabajo.

\section{BiBLIOGRAFÍA}

Albanesi, A; CR Kunst; AL Anriquez; JE Silverman; R LEDESMA; ET AL. 2013. Rolado selectivo de baja intensidad (RBI) y sistemas silvopastoriles de la región chaqueña. Pp. 147-174 en: Albanesi, A; R Paz; MT Sobrero; S Helman \& S Rodríguez (eds.). Hacia la Construcción del Desarrollo Agropecuario y Agroindustrial. De La FAyA Al NOA. Ediciones Magna. Tucumán.

Anderson, MJ; TO Crist; JM Chase; M Vellend; BD InOuYe; ET AL. 2011. Navigating the multiple meanings of $\beta$ diversity: a roadmap for the practicing ecologist. Ecol. Lett., 14:19-28.

Areskoug, V. 2001. Utilisation of remnant dry-forest corridors by the native fauna in a pastural landscape in the Paraguayan Chaco. CBMs Skr., 3:25-38.

BIONDI, LM; MS Bó \& M FAVERO. 2005. Diet of the Chimango Caracara (Milvago chimango) during the breeding season in the southeastern Buenos Aires Province, Argentina. Ornitol. Neotropical, 16:31-42.

Boletta, P. 1998. Clima. Pp. 7-21 en: Casas, R (ed.). Desmonte y Habilitación de Tierras en la Región Chaqueña Semiárida. FAO. Santiago, Chile.

BonHAm, CD. 1989. Measurements for Terrestrial Vegetation. Wiley.

BUCHER, EH. 1980. Ecología de la fauna chaqueña: una revisión. ECOSUR, 7:111-159.

BuCHER, EH. 1982. Chaco and Caatinga - South American Arid Savannas, Woodlands and Thickets. Pp. 48-79 en: Huntley, BJ \& BH Walker (eds.). Ecology of Tropical Savannas. SpringerVerlag. Berlin.

CANavelli, SB. 2010. Consideraciones de manejo para disminuir los daños por aves en girasol. Publicación Miscelánea (No. 118). INTA - Estación Experimental Agropecuaria Rafaela. Santa Fe, Argentina.

Codesido, M \& D Bilenca. 2004. Variación estacional de un ensamble de aves en un bosque subtropical semiárido del Chaco Argentino. BIOTROPICA, 36:544-554.

Codesido, M \& D Bilenca. 2000. Comparación de los métodos de transectas de faja y de conteo de puntos de radio fijo en una comunidad de aves del bosque semiárido santiagueño. Hornero, 15:85-91.

Codesido, M; PGADO; A DroZd \& D Bilenca. 2009. Respuestas 
de un ensamble de aves a la remoción manual de arbustos en un bosque subtropical semiárido del Chaco Argentino. Ornitol. Neotropical, 20:47-60.

CORIA, OR. 2014. Evaluación de tres modelos de la Teoría de Metacomunidades. Un estudio de caso con las comunidades de aves de la Sierra de Guasayán (Santiago del Estero, Argentina). Tesis doctoral. Facultad de Ciencias Forestales, Universidad Nacional de Santiago del Estero.

CORIA, RD; OR CORIA \& CR Kunst. 2012. Rolado Selectivo de Baja Intensidad (RBI) y aves en un bosque del Chaco Semiárido. Presentado en el $2^{\circ}$ Congreso Nacional de Sistemas Silvopastoriles. INTA. Santiago del Estero.

Cueto, VR. 1996. Relación entre los ensambles de aves y la estructura de la vegetación: Un análisis a tres escalas espaciales. Tesis doctoral. Facultad de Ciencias Exactas y Naturales, Universidad de Buenos Aires.

Di Giacomo, AG. 2005. Aves de la Reserva El Bagual. Pp. 201-465 en: Di Giacomo, AG \& SF Krapovickas (eds.). Historia Natural y Paisaje de la Reserva El Bagual, Formosa, Argentina. Inventario de La Fauna de Vertebrados y de La Flora Vascular de un Area del Chaco Húmedo. Temas de Naturaleza y Conservación 4:1-592. Aves Argentinas / Asociación Ornitológica del Plata. Buenos Aires.

Di Rienzo, JA; F CASANoves; MG Balzarini; L GonzÁlez; M TABLADA; ET AL. 2013. Infostat versión 2013. Grupo Infostat, FCA, Universidad Nacional de Córdoba. Argentina.

FernÁndez, E; EV Álvarez \& MB Martella. 1997. Variación estacional de la abundancia poblacional del Calancate Común (Aratinga acuticaudata) en la Reserva Chancaní, Córdoba, Argentina. Hornero, 14:259-262.

FISCHER, J; DB LINDENMAYER \&AD MANNING. 2006. Biodiversity, ecosystem function, and resilience: ten guiding principles for commodity production landscapes. Front. Ecol. Environ., 4: 80-86.

Fraga, R \& T NARosky. 1985. Nidificación de las aves argentinas (Formicariidae a Cinclidae). Asociación Ornitológica del Plata. Buenos Aires. Argentina.

Galmes, MA; MA SantilláN; MM Reyes \& JH Sarasola. 2008. Variaciones estacionales en la dieta del halconcito gris (Spiziapteryx circumcinctus) en el centro de Argentina. Presentado en la XII Reunión Argentina de Ornitología. San Martín de los Andes, Neuquén.

GibBons, DW; DA Hill \& WJ Sutherland. 1996. Birds. [8] Pp. 227-259 en: Sutherland, WJ (ed.). Ecological Census Techniques. A Handbook. Cambridge University Press.

Giraudo, L; M Kufner; R Torres; D TAmburini; V Briguera; ET AL. 2006. Avifauna del bosque chaqueño oriental de la provincia de Córdoba, Argentina. Ecol. Appl., 5:127136.

HiLl, MO \& HGJ GAUCH. 1980. Detrended correspondence analysis: An improved ordination technique. Vegetatio, 42:47-58.

Instituto de Botánica Darwinion. 2014. Flora Argentina. Plantas Vasculares de la República Argentina. http:// www.floraargentina.edu.ar (acceso 20/08/14).

INTA EEA Santiago del Estero. 2014. Agrometeorología Santiago Estero. PRODAGRO. http:/ / meteosgo.inta.ar/ Meteo/Default.aspx (acceso 14/10/14).

KENT, M \& P COKER. 1992. Vegetation description and analysis: a practical approach. CRC Press, Inc., 2000 Corporate Blvd. N.W. Boca Raton, Florida.

Kunst, CR; R Ledesma; S Bravo; A Albanesi \& J Godoy.
2012. Rolados y diversidad botánica II: efecto en el sitio ecológico alto. Presentado en el $2^{\circ}$ Congreso Nacional de Sistemas Silvopastoriles. INTA. Santiago del Estero.

Kunst, CR; R Ledesma \& M Navall (eds.). 2008. RBI: Rolado Selectivo de Baja Intensidad. INTA. Santiago del Estero.

LEPŠ, J \& T ŠMILAUER. 2003. Multivariate analysis of ecological data using CANOCO. Cambridge University Press. Cambridge, UK.

Lopez de Casenave, J; JP Pelotto; SM Caziani; M Mermoz \& J PROTOMASTRO. 1998. Responses of avian assemblages to a natural edge in a Chaco Semiarid forest in Argentina. The Auk, 115:425-435.

LOPEZ DE CASENAVE, J. 2001. Estructura gremial y organización de un ensamble de aves del Desierto del Monte. Tesis doctoral. Facultad de Ciencias Exactas y Naturales, Universidad de Buenos Aires.

Macchi, L; HR Grau; PV Zelaya \& S Marinaro. 2013. Trade-offs between land use intensity and avian biodiversity in the dry Chaco of Argentina: A tale of two gradients. Agric. Ecosyst. Environ., 174:11-20.

Martella, MB; EV Trumper; LM Bellis; D Renison; PF GIORDANO; ET AL. 2012. Manual de Ecología. Evaluación de la biodiversidad. REDUCA, 5(1):71-115.

Mastrangelo, ME. 2014. Conservation on the Frontier: Understanding and influencing how cattle production impacts avian diversity in the Dry Chaco Forests of Argentina. Doctoral thesis. Victoria University of Wellington.

Mastrangelo, ME \& MC Gavin. 2012. Trade-offs between cattle production and bird conservation in an agricultural frontier of the Gran Chaco of Argentina. Conserv. Biol., 26:1040-1051.

MASTRANGELO, ME \& M GAVIN. 2014. Impacts of agricultural intensification on avian richness at multiple scales in Dry Chaco forests. Biol. Conserv., 179:63-71.

Milesi, FA; L Marone; J Lopez de Casenave; VR Cueto \& ET MEZQuidA. 2002. Gremios de manejo como indicadores de las condiciones del ambiente: un estudio de caso con aves y perturbaciones del hábitat en el Monte central, Argentina. Ecol. Austral, 12:149-161.

Morello, J \& J Adamoli. 1968. Las grandes unidades de vegetación y ambiente del Chaco argentino. Primera parte: objetivos y metodología. Serie Fitogeográfica No 10. INTA.

Morello, J \& CA Saravia Toledo. 1959. El Bosque Chaqueño I. Paisaje primitivo, paisaje natural y paisaje cultural en el oriente de Salta. Rev. Agronómica Noroeste Argent., 3:5-81.

Moreno, CE. 2001. Métodos para medir la biodiversidad. M\&T-Manuales y Tesis SEA. Zaragoza.

Remsen, JV Jr; CD Cadena; A Jaramillo; M Nores; JF PACHECO; ET AL. 2014. A Classification of the bird species of South America. American Ornithologists' Union. http:/ /www.museum.lsu.edu/ Remsen/SACCBaseline.htm (acceso 20/08/14).

RINEY, T. 1982. Study and management of large mammals. Wiley.

Ter BraAk, CJ \& P Šmilauer. 2002. CANOCO Reference Manual and CanoDraw for Windows User's Guide: Software for Canonical Community Ordination (Version 4.5). Microcomputer Power. Ithaca, NY, USA.

ZAR, JH. 1999. Biostatistical analysis. Prentice Hall PTR. 